\title{
Macroscopic quantum Schrödinger and Einstein-Podolsky-Rosen paradoxes
}

\author{
M. D. REID* and E. G. CAVALCANTI \\ Physics Department, The University of Queensland, \\ Brisbane, Australia
}

(Received 15 February 2005; in final form 10 May 2005)

\begin{abstract}
We propose macroscopic generalizations of the Einstein-Podolsky-Rosen paradox in which the completeness of quantum mechanics is contrasted with forms of macroscopic reality and macroscopic local reality defined in relation to Schrödinger's original 'cat' paradox.
\end{abstract}

\section{Introduction}

In his famous 1935 essay, Schrödinger [1] discussed how 'ridiculous' it would be to set up a case where a cat would be described by a superposition of dead and alive states. The underlying assumption in Schrödinger's remark is what we might call 'macroscopic realism'. This premise states that, even if we accept that microscopic systems do not possess predetermined values for all physical quantities, as quantum mechanics tells us, there can be no such lack of a macroscopic predetermined value, so that the cat must be dead or alive.

Leggett [2] discussed the possibility of tests of the existence of macroscopic superpositions. He introduced the idea of macroscopic variables, whose only essential characteristic is that 'appreciably' different values of the variable should correspond to macroscopically distinguishable states. In some cases, indeed, two states can be considered macroscopically distinguishable even when the number of particles is not strictly macroscopic. This view has in fact become increasingly frequent in the literature, as pointed out, for example, by Laloë [3].

The work of Leggett and Garg [4] addressed the fundamental issue of proving an incompatibility of quantum mechanics with the premise of 'macroscopic realism' defined in conjunction with a 'macroscopic non-invasive measurability'. This work illustrates the strength and simplicity of the fundamental approach along the lines considered by Bell [5], in which predictions of quantum mechanics are shown to disagree with those of well-defined classical premises, so that an experiment

\footnotetext{
*Corresponding author. Email: margaret@physics.uq.edu.au
} 
could in principle be performed to directly refute those classical premises, without invoking assumptions based on the correctness of quantum mechanics.

With this in mind, we point out that the proof of a macroscopic quantum superposition or entanglement may not be enough to prove a failure of a 'macroscopic reality' as defined for example in the sense of Leggett and Garg, in the same way that the proof of entanglement is not generally enough to disprove the local hidden variable theories ('local realism') considered by Bell.

In this paper we therefore take several criteria that can be shown to be signatures of macroscopic (entangled) superpositions, and explicitly discuss the extent to which we can claim an incompatibility with the premise of 'macroscopic realism' (or a 'macroscopic local reality'). In doing so, we realize that we cannot prove a falsification of macroscopic realism (macroscopic local reality) directly, but we can prove a macroscopic Einstein-Podolsky-Rosen (EPR) paradox [6] in which a 'macroscopic reality' ('macroscopic local reality') is found to be inconsistent with the completeness of quantum mechanics.

\section{Some criteria for macroscopic superpositions}

We first summarize criteria that can be used in the case of squeezed states [7] to detect macroscopic superpositions with respect to the basis states associated with a macroscopic variable.

Suppose that we have two subsystems $A$ and $B$. Suppose that the results of a measurement of an observable $\hat{O}$ (say, position $\hat{x}$ ) performed at $A$ can be mapped onto two possible and distinct sets of outcomes, designated by $\lambda_{c}=-1$ or $\lambda_{c}=1$. The outcomes are macroscopically distinct in the values of the observable $\hat{O}$ when the regions $\lambda_{c}=-1$ ('dead') and $\lambda_{c}=1$ ('alive') are macroscopically different in $x$ so that there is zero probability for a result in a middle region (figure 1).

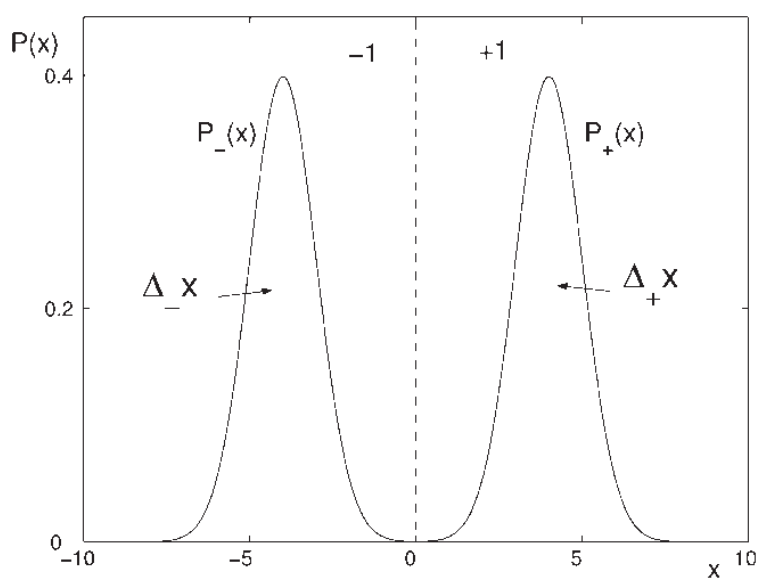

Figure 1. Probability distribution for measurement $\hat{x}$ which gives two macroscopically separated outcomes $\lambda_{c}= \pm$. 
The probability distribution for $x$ becomes $P(x)=P_{-} P_{-}(x)+P_{+} P_{+}(x)$ where $P_{ \pm}(x)$ is the distribution (with variance $\left.\Delta_{ \pm}^{2} x\right)$ for $x$, given that the result indicates $\lambda_{c}= \pm 1$.

Any density operator for the composite system can be written in terms of the basis states $\left|o_{i}\right\rangle_{A}$ of $\hat{O}$ and $\left|o_{r}\right\rangle_{B}$ of an observable $\hat{O}^{B}$ of $B$ as

$$
\rho=\sum_{R} P_{R}\left|\psi_{R}\right\rangle\left\langle\psi_{R}\right|
$$

where the pure states are

$$
\left|\psi_{R}\right\rangle=\sum_{i, r} c_{i, r}^{R}\left|o_{i}\right\rangle_{A}\left|o_{r}\right\rangle_{B}
$$

To prove that the system exists in the superposition state $\left|o_{i}\right\rangle_{A}\left|o_{p}\right\rangle_{B}+\left|o_{j}\right\rangle_{A}\left|o_{q}\right\rangle_{B}$ (where $o_{i}$ and $o_{j}$ are meso- or macroscopically different) with some non-zero probability, we need to prove that for at least one of the $R$ with non-zero $P_{R}$, there must be a superposition of the type

$$
\left|\psi_{R}\right\rangle=c_{+}^{R}\left|\psi_{+}\right\rangle+c_{-}^{R}\left|\psi_{-}\right\rangle,
$$

where $\left|\psi_{+}\right\rangle=\sum_{o_{i} \in \lambda_{+}, r} c_{i, r}^{R}\left|o_{i}\right\rangle_{A}\left|o_{r}\right\rangle_{B}$ and $\left|\psi_{-}\right\rangle=\sum_{o_{j} \in \lambda_{-}, s} c_{j, s}^{R}\left|o_{j}\right\rangle_{A}\left|o_{s}\right\rangle_{B}$ and it being the case that at least one of the $c_{i, r}^{R}$ is non-zero (for $r=p$, say), and also one of the $c_{j, s}^{R}$ is non-zero (for $s=q$, say). (Here $o_{i} \in \lambda_{+}$means $o_{i}$ gives a result $\lambda_{c}=+1$.)

If the macroscopic superposition state (3) does not appear in the expansion (1) then the density operator is expressible as

$$
\rho_{\text {mix }}=P_{+}^{0} \rho_{+}+P_{-}^{0} \rho_{-}
$$

where the only restriction placed on the density operator $\rho_{ \pm}$is that the prediction for the result of the measurement $\hat{x}$ say at $A$ is to always give, respectively, $\lambda_{c}= \pm 1$. This follows because we are able to express each component pure state $\left|\psi_{R}\right\rangle$ as either one of $\left|\psi_{+}\right\rangle$or $\left|\psi_{-}\right\rangle$.

We summarize the following theorem [8].

Theorem 1: Where the position $x$ of system $A$ distinguishes $\lambda_{c}= \pm 1$, we can derive straightforwardly from the assumption (4) the relation

$$
\Delta_{\text {ave }} x \Delta_{\text {inf }} p \geq 1
$$

The proof, and extensions applying to spin measurements, have been presented elsewhere [7], but are based on the assumption that the individual $\rho_{ \pm}$are quantum densities and satisfy the uncertainty relation $\Delta x \Delta p \geq 1$. We define the measurable variances $\Delta_{I}^{2} x$ of $P_{I}(x)(I= \pm 1)$ and their average $\Delta_{\text {ave }}^{2} x=\sum_{I} P_{I} \Delta_{I}^{2} x$. We define an average inference variance $\Delta_{\text {inf }}^{2} p=\sum_{p^{B}} P\left(p^{B}\right) V\left[p \mid p^{B}\right]: V\left[p \mid p^{B}\right]$ is the variance of conditional distribution $P\left(p \mid p^{B}\right)$ for the result $p$ at $A$, given a result $p^{B}$ for a measurement at $B$, and $P\left(p^{B}\right)$ is the probability of $p^{B}$.

Violation of the inequality (5) then implies the superposition (3). 


\section{Criterion for macroscopic entanglement}

We present a new criterion which will enable proof of a macroscopic entanglement. To prove a macroscopic entanglement [1] we want to prove the existence of the state (3) where $p \neq q$, in the density matrix expansion (1), and we prove below a sufficient condition for such a state.

If such a macroscopic entangled state does not appear in (1) we are able to express each component pure state $\left|\psi_{R}\right\rangle$ as either one of $\left|\psi_{+}\right\rangle$or $\left|\psi_{-}\right\rangle$, or as a separable state of type $\left\{\sum_{m_{i} \in \lambda_{+}} c_{i, r}^{R}\left|m_{i}\right\rangle+\sum_{m_{j} \in \lambda_{-}} c_{j, r}^{R}\left|m_{j}\right\rangle\right\}_{A}\left|m_{r}\right\rangle_{B}$. In this case the density operator is expressible as

$$
\rho=P_{m} \rho_{\text {mix }}+P_{s} \rho_{\text {sep }}
$$

Here $\rho_{\text {mix }}$ represents any quantum mixture of the form (4) and $\rho_{\text {sep }}$ represents any separable density operator expressible as $\rho_{\text {sep }}=\sum_{l} P_{l} \rho_{l}^{A} \rho_{l}^{B}$, where $\rho_{l}^{A}$ and $\rho_{l}^{B}$ are density operators for systems $A$ and $B$ respectively.

Theorem 2: Where one has maximum correlation between +1 and -1 outcomes at $A$ and a measurement at $B$, so that we can infer with certainty the \pm result at $A$ by measuring at $B$, then the assumption that the density operator is of the form (6) will imply inequality (5). The violation of these constraints in conjunction with proof of correlation will therefore imply the existence of macroscopic entanglement.

Proof: Firstly we note that for a system described by $\rho_{\text {mix }}$ the inequality (5) holds, as proved by Theorem 1. The correlation implies the conditional probability $P\left( \pm \mid O_{i}^{B}\right)$ for an outcome in the set $\lambda= \pm 1$ at $A$ given a result $O_{i}^{B}$ for a measurement at $B$ will either be 0 or 1 . For a separable system $\rho_{\text {sep }}$

$$
\begin{aligned}
P\left( \pm \mid O_{i}^{B}\right) & =\frac{P\left( \pm \cap O_{i}^{B}\right)}{P\left(O_{i}^{B}\right)} \\
& =\frac{\sum_{l} P_{l} P_{l}^{A}( \pm) P_{l}^{B}\left(O_{i}^{B}\right)}{\sum_{l} P_{l} P_{l}^{B}\left(O_{i}^{B}\right)}
\end{aligned}
$$

so that if $P\left( \pm \mid O_{i}^{B}\right)=1$, then for each $l$, either $P_{l}^{A}( \pm)=1$, in which case we label $l$ with a + , or $P_{l}\left(O_{i}^{B}\right)=0$, in which case we label $l$ with a -1 , since it must be the case that here the outcome for $A$ would be -1 . With this we see that the expansion for $\rho_{\text {sep }}$ takes the form of a mixture of + and - quantum states for $A$. If this is the case, then the density operator of (6) can be written in the form of $\rho_{\text {mix }}$ and therefore the constraint (5) follows.

\section{Macroscopic superpositions in squeezed entangled states}

We consider the Gaussian EPR states which are of considerable experimental interest [9-14], and for which entanglement, at least a microscopic entanglement, has been confirmed experimentally. An optimal theoretical quantum model 
is the two-mode squeezed state [8, 15-17]

$$
|\psi\rangle=\sum_{n=0}^{\infty} c_{n}|n\rangle_{A}|n\rangle_{B}
$$

where $c_{n}=\tanh ^{n} r / \cosh r$ and $|n\rangle_{A}$ and $|n\rangle_{B}$ are the number states for the fields at $A$ and $B$, which have boson operators $\hat{a}$ and $\hat{b}$ respectively. Measurements are of the amplitudes: $\hat{x}=\left(\hat{a}^{\dagger}+\hat{a}\right), \quad \hat{p}=i\left(\hat{a}^{\dagger}-\hat{a}\right), \quad \hat{x}^{B}=\left(\hat{b}^{\dagger}+\hat{b}\right), \quad \hat{p}^{B}=i\left(\hat{b}^{\dagger}-\hat{b}\right) \quad$ where $\Delta^{2} x \Delta^{2} p \geq 1$. The results of an amplitude measurement $\hat{x}$ are classified as $\lambda_{c}=+1$ if $x \geq 0$, and $\lambda_{c}=-1$ if $x<0$.

The probability distribution (figure 2) for $x$ is the Gaussian

$$
P(x)=\frac{1}{\sqrt{2 \pi \sigma}} e^{-x^{2} / 2 \sigma}
$$

where $\sigma=\cosh 2 r$. As the squeeze parameter $r$ increases, this variance increases, the system becoming macroscopic in amplitude and photon number. The probability distribution for $x$ where $\lambda_{c}= \pm$ is the half-Gaussian. This gives $\Delta_{\text {ave }}^{2} x=0.36 \sigma$. The two-mode squeezed state predicts [15-17] for infinite $r$ the EPR correlation $x=x^{B}$, and $p=-p^{B}$. For arbitrary $r V\left[x \mid x^{B}\right]=V\left[p \mid p^{B}\right]=1 / \cosh 2 r$ for all $x^{B}, p^{B}$. The result for measurement $\hat{p}_{a}$ at $A$ may be inferred from a measurement $\hat{p}^{B}$ at $B$, but the average inference variance is $\Delta_{\text {inf }}^{2} p=1 / \cosh 2 r$. This gives $\Delta_{\text {inf }}^{2} p \Delta_{\text {ave }}^{2} x=0.36$.

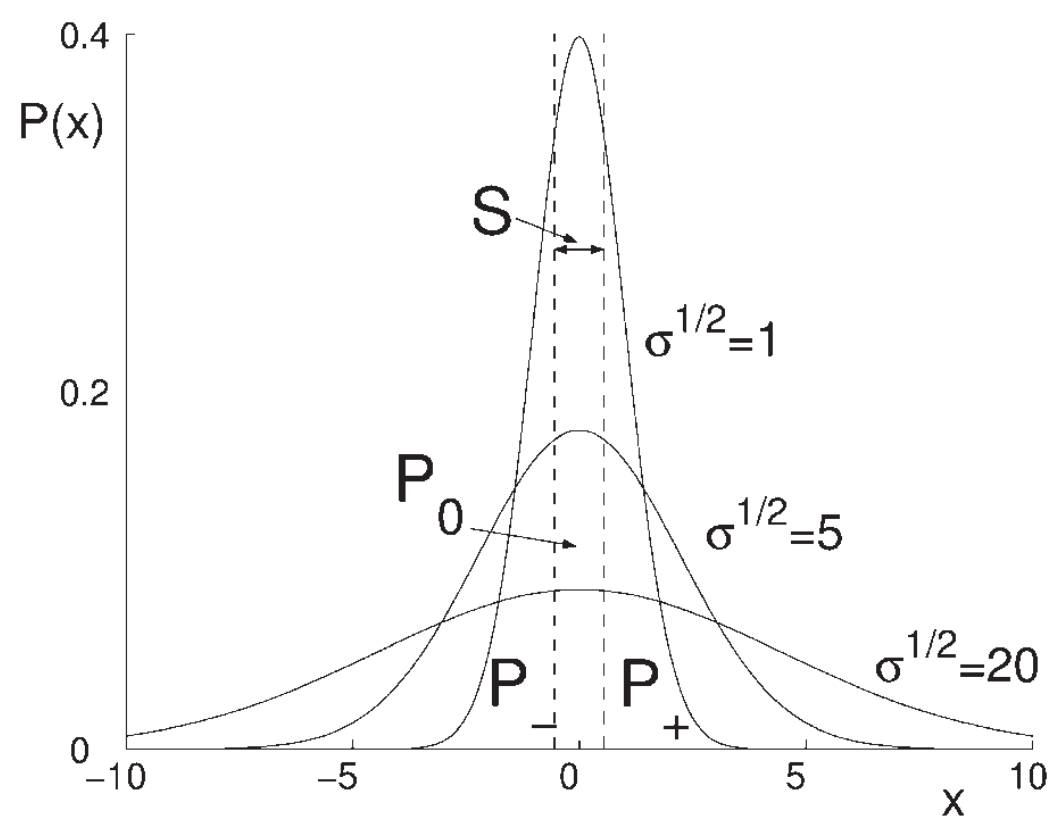

Figure 2. Probability distribution for measurement $x$ for a two-mode or simple one-mode squeezed state. For fixed $S$, no matter how large, there is a vanishing probability $P_{o}$, for $-S / 2<x<S / 2$, as the variance $\sigma$ increases. This indicates that in the limit of infinite $\sigma$ the outcomes + and - are macroscopically distinct. 
As $r$ becomes large, the outcomes $\lambda_{c}=+1$ and $\lambda_{c}=-1$ become macroscopically distinct [8] (see argument given in figure 2). Since here the limiting value of $\Delta_{\text {inf }} p \Delta_{\text {ave }} x$ is distinctly different to (and less than) one, there is a macroscopic superposition of type (3): the system cannot be in a mixture of the type (4) (Theorem 1). It is also possible to prove a macroscopic entanglement (Theorem 2). As $r$ increases, the result $x_{i}^{B}$ of measurement $\hat{x}^{B}$ at $B$ will (absolutely) imply the result $x=x_{i}^{B}$ for $\hat{x}$ at $A$ with no error, and similarly the result $p^{B}$ at $B$ will imply the result $p=-p^{B}$ at $A$; we have perfect EPR correlations [15-17] and a macroscopic entanglement.

\section{Macroscopic paradoxes}

\subsection{Schrödinger's reality and EPR}

While the signature of a macroscopic superposition or entanglement proves the impossibility of the macroscopic system being in any mixture (4) of quantum states $\rho_{+}$and $\rho_{-}$

$$
\rho_{\text {mix }}^{A B}=P_{+} \rho_{+}^{A B}+P_{-} \rho_{-}^{A B}
$$

it does not exclude mixtures where the components are hidden variable states, as considered by Bell [5]. This is the essence of an EPR-type argument [6], where the assumption of a form of reality (in this case Schrödinger's 'macroscopic reality') gives an argument for the 'completion' (hidden variable interpretation) of quantum mechanics.

\subsection{Direct macroscopic EPR paradox for entangled systems}

The bipartite entangled systems, where we satisfy conditions for macroscopic entanglement as given by Theorem 2, allow a more direct macroscopic example of the EPR paradox, if the two subsystems $A$ and $B$ are spatially separated. For the infinite $r$, the measurement at $B$ implies with certainty either the 'alive' or 'dead' result at $A$. The EPR premises of 'local realism' (here, the reader is referred to previous papers of EPR [6] and Mermin [18]) lead to the conclusion that the state of the 'cat' is predetermined to be 'alive' or 'dead' prior to its measurement. This predetermined state of the cat may be represented by a macroscopic hidden variable (or 'element of reality') $\lambda_{1}: \lambda_{1}=1$ implies the predetermined state of 'alive'; $\lambda_{1}=-1$ implies the predetermined state of 'dead'. The variance of the conditional distribution $P(x \mid+1)$ for the result $x$ at $A$, given that the measurement at $B$ implies the +1 outcome at $A$, is $\Delta_{+}^{2} x$ and this gives the degree of definiteness, in the prediction of $x$, associated with the hidden variable $\lambda_{1}=1$; similarly $\Delta_{-}^{2} x$. Note that $\Delta_{\text {inf }}^{2} x$ (defined similarly to $\Delta_{\text {inf }}^{2} p$ of theorem 2 ) is actually equal to $\Delta_{\text {ave }}^{2} x$.

There is also a correlation between the momenta of the separated subsystems. The EPR conclusion then is that the 'cat' system at $A$ is also described by a hidden variable $\lambda_{2}$, to predetermine the result for momentum. The prediction for the momentum measurement at $A$ given a measurement at $B$ is narrow, so that $\Delta_{\text {inf }} x \Delta_{\text {inf }} p=\Delta_{\text {ave }} x \Delta_{\text {inf }} p<1$. The state of the cat as given by the hidden variables 
$\lambda_{1}$ and $\lambda_{2}$ defines momentum and position more definitely than can be given by a quantum state, and this is the situation of the EPR paradox [15-17], that 'local realism' can only be consistent with the correlations of this system through a 'completion' of quantum mechanics, where non-quantum hidden variable states are introduced to represent the 'dead' and 'alive' states so that there can be a realization of some sort of mixture.

The violation of (5) here however is a strong EPR paradox, because the 'dead' and 'alive' descriptions are macroscopically distinct, and the EPR conclusion that the system is described by an 'element of reality' $\lambda_{1}$ is further justified by Schrödinger's 'macroscopic reality'.

\subsection{Gaussian EPR states}

The violation of (5) for the two-mode squeezed state (7) could be predicted by a mixture, where the 'cat' is in a hidden variable state $\phi(x, p$,$) . Such a hidden variable$ mixture is perfectly consistent with the notion of the 'cat' being 'dead' or 'alive'. The Wigner function $W(x, p$,$) , being positive for the two-mode squeezed state, provides$ the probability density for the hidden variable state of the 'cat', where we are able to make the correspondence $\lambda_{1}=x /|x|$ and $\lambda_{2}=p$, these being the predetermined results of the $x$ and $p$ measurements. This description of the system as a hidden variable mixture, however, cannot correspond to a quantum mixture, because of the definiteness of the prediction of the measurements for $x$ and $p$ that is required of the hidden variable states.

\section{Conclusion}

Violation of certain inequalities may be sufficient to prove a macroscopic (entangled) superposition, so that the system cannot be described as a mixture of dead and alive quantum states, but we cannot exclude the possibility of hidden variable mixtures which give a consistency with Schrödinger's and Leggett's 'macroscopic realism'.

\section{References}

[1] E. Schrödinger, Naturwissenschaften 23807 (1935).

[2] A.J. Leggett, Contemp. Phys. 25583 (1984).

[3] F. Laloë, Am. J. Phys. 69655 (2001).

[4] A.J. Leggett and A. Garg, Phys. Rev. Lett. 54857 (1985).

[5] J.S. Bell, Physics. 1195 (1965).

[6] A. Einstein, B. Podolsky and N. Rosen, Phys. Rev. 47777 (1935).

[7] C.M. Caves and B.L. Schumaker, Phys. Rev. A 313068 (1985).

[8] The result follows by noting that the uncertainty relation holds for each of $\rho_{+}$and $\rho_{-}$, and applying the Cauchy-Schwarz inequality.

[9] Z.Y. Ou, et al., Phys. Rev. Lett. 683663 (1992).

[10] Y. Zhang, et al., Phys. Rev. A 62023813 (2000).

[11] C. Silberhorn, et al., Phys Rev. Lett. 864267 (2001). 
[12] W. Bowen, et al., Phys. Rev. Lett. 90043601 (2003).

[13] E. Giacobino and C. Fabre, Appl. Phys. B 55189 (1992).

[14] C. Schori, et al., Phys. Rev. A 66033802 (2002).

[15] M.D. Reid, Phys. Rev. A 40913 (1989).

[16] M.D. Reid, quant-ph/0103142.

[17] M.D. Reid and P.D. Drummond, Phys. Rev. Lett. 602731 (1988).

[18] N.D. Mermin, Phys. Today 439 (1990). 